\title{
Program for Implementing Software Quality Metrics
}

by H.P. Yule and C.A. Rierner

Advanced Computer Applications Center,

Environmental Assessment and Information Sciences Division,

Argonne National Laboratory, 9700 South Cass Avenue, Argonne, Illinois 60439

April 1992

Work sponsored by Department of Veterans Affairs, Veterans Benefits Administration 


\section{CONTENTS}

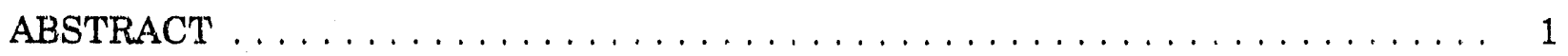

1 INTRODUCTION $\ldots \ldots \ldots \ldots \ldots \ldots \ldots \ldots \ldots \ldots \ldots \ldots \ldots$

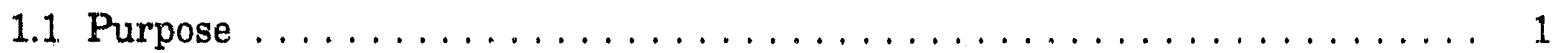

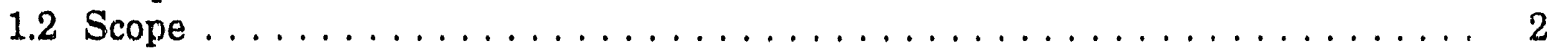

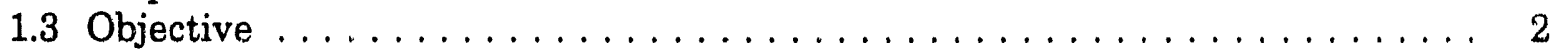

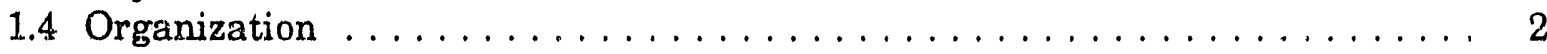

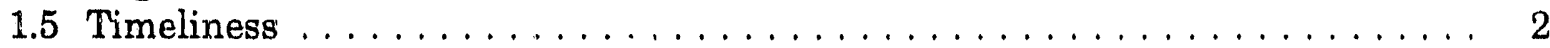

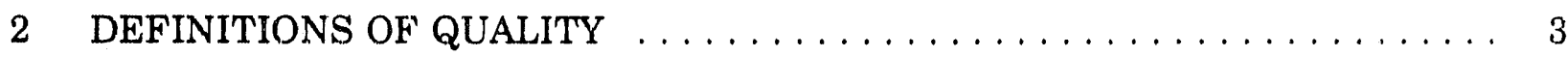

2.1 Quality in General .............................. 3

2.2 Quality with Respect to Software $\ldots \ldots \ldots \ldots \ldots \ldots \ldots \ldots \ldots \ldots$

3 REQUIREMENTS FOR SUCCESSFUL METRICS IMPLEMENTATION $\ldots \ldots \ldots$

3.1 General Requirements $\ldots \ldots \ldots \ldots \ldots \ldots \ldots \ldots \ldots \ldots \ldots \ldots$

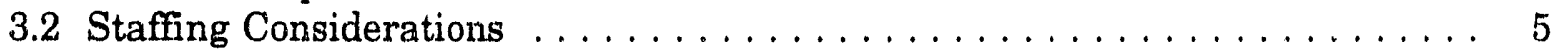

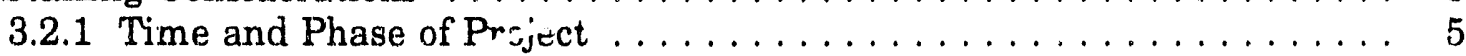

3.2.2 Management of Personnel $\ldots \ldots \ldots \ldots \ldots \ldots \ldots \ldots \ldots$

4 FACTORS THAT REVEAL SOFTWARE QUALITY $\ldots \ldots \ldots \ldots \ldots \ldots \ldots$

5 SOFTWARE QUALITY METRICS $\ldots \ldots \ldots \ldots \ldots \ldots \ldots \ldots \ldots \ldots$

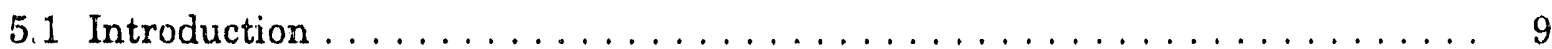

5.2 Resource (Cost) Metrics . . . . . . . . . . . . . . . . . . . . . . . 10

5.3 Software Design Metrics $\ldots \ldots \ldots \ldots \ldots \ldots \ldots \ldots \ldots \ldots \ldots \ldots$

5.4 Software Product Metrics for Code Being Maintained . . . . . . . . . . . 14

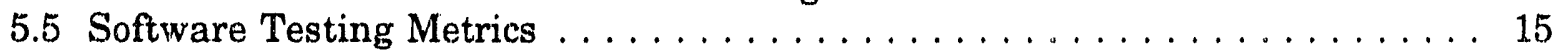

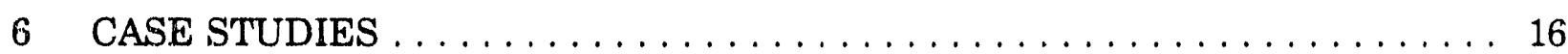

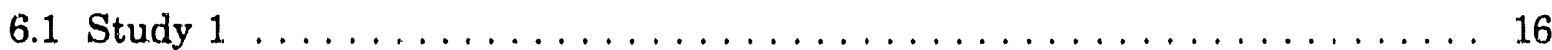

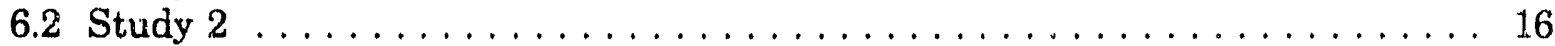

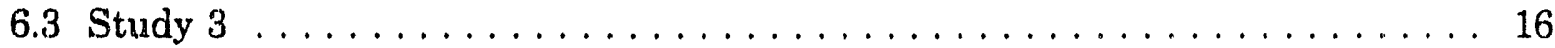

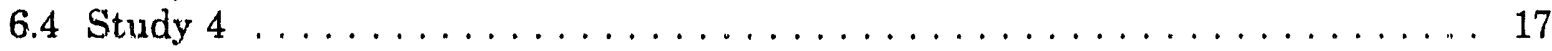

7 SUGGESTED METRICS TO BENEFIT 'THE VETERANS BENEFITS ADMINISTRATION $\ldots \ldots \ldots \ldots \ldots \ldots \ldots \ldots \ldots \ldots \ldots$

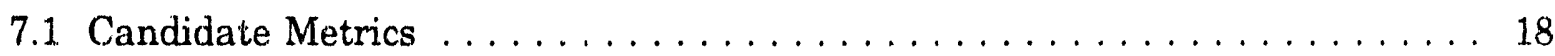

7.1 .1 Software Design Metrics $\ldots \ldots \ldots \ldots \ldots \ldots \ldots \ldots \ldots \ldots$

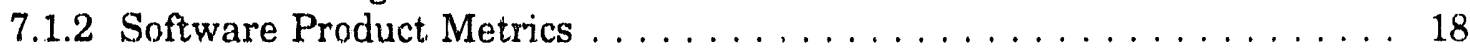

7.1 .3 Software Testing Metrics $\ldots \ldots \ldots \ldots \ldots \ldots \ldots \ldots \ldots \ldots \ldots$

7.1 .4 Resource Metrics . . . . . . . . . . . . . . . . . . . . 19 


\section{CONTENTS (Cont'd)}

7.2 Processing the Acquired Metric Data ..................... 20

7.2.1 Step 1: Analyze the Metric Data ................... 20

7.2.2 Step 2: Validate the Analysis $\ldots \ldots \ldots \ldots \ldots \ldots \ldots \ldots \ldots, 20$

8 IMPLEMENTATION PLAN $\ldots \ldots \ldots \ldots \ldots \ldots \ldots \ldots \ldots \ldots \ldots \ldots \ldots$

\section{FIGURES}

1 Staffing Level as a Function of Project Phase and Time $\ldots \ldots \ldots \ldots \ldots \ldots$

2 Error Rate as a Function of Software System Complexity . . . . . . . . . . . 12

3 Error Rate as a Function of Module Size $\ldots \ldots \ldots \ldots \ldots \ldots \ldots \ldots \ldots \ldots$

\section{TABLE}

1 Suggested Contents for Problem Report $\ldots \ldots \ldots \ldots \ldots \ldots \ldots \ldots \ldots \ldots$ 


\title{
PROGRAM FOR IMPLEMENTING SOFTWARE QUALI'TY METRICS
}

by

H.P. Yule and C.A. Riemer

\begin{abstract}
This report describes a program by which the Veterans Benefit Administration (VBA) can implement metries to measure the performance of automated data systems and demonstrate that they are improving over time. It provides a definition of quality, particularly with regard to software. Requirements for management and staff to achieve a successful metrics program are discussed. It lists the attributes of high-quality software, then describes the $m$ strics or calculations that can be used to measure these attributes in a particular system. Case studies of some successful metrics programs used by business are presented. The report ends with suggestions on which metrics the VBA should use and the order in which they should be implemented.
\end{abstract}

\section{INTRODUCTION}

\subsection{PURPOSE}

The Veterans Benefits Administr-t.on (VBA) has written a description of the software development life cycle (SDLC) and established guidelines designed to result in the orderly and controlled development and maintenance of software." Within the VBA, the Systems Development Service (SDS) is charged with the design and development of the VBA's automated data systems and with providing ongoing support for them. The goal of SDS is to improve the development and maintenance of software for VBA system users. This document provides SDS with a plan for implementing software metrics as a way to determine its success in approaching these goals. (A metric is a calculation or composite indicator that is determined on the basis of twn or more measures. The software metrics described here are calculations of measures or attributes that reveal the quality of a software program or system.)

\footnotetext{
"VBA, 1992, The Veterans Benefits Administration: System Development Life Cycle Process Description and Gui 'eline Manual, Department of Veterans Affairs, Washington, D.C.
} 


\subsection{SCOPE}

This document (1) defines both quality in general and software quality in particular, (2) lists requirements for implementing a successful metrics program, (3) reviews suftware quality factors, and (4) reviews available software quality metrics to provide information on software quality factors. Sorne examples of what is being done in the industrial sector are provided as background material, and recommended steps for implementation of software metrics by the VBA are provided.

\subsection{OBJECTIVE}

The goal of this document is to provide the VBA with a plan for a workable, effective, and viable method of implementing software metrics to measure software performance and demonstrate that the software system is improving with time.

\subsection{ORGANIZATION}

Section 2 gives a basic, general definition of quality as it applies to organized activities such as those conducted by industry, the government, and all kinds of businesses. It also provides an overview of definitions of quality as it applies to software. Section 3 presents a brief discussion of staffing requirements, considerations, and caveats related to a metrics implementation program. It discusses those management practices that are needed to provide a solid foundation for implementing software metrics. Section 4 presents a list of software factors that can be used to evaluate software quality. These factors (or attributes), when taken together, provide an adequate measure of software quality. Section 5 describes the metrics that provide information on the software quality factors of Section 4 . The analysis of data provided by the metrics will be the basis for corrective actions and further improvements. Section 6 contains examples of some metrics employed by industry and business for process and product improvement. Section 7 is a list of suggested metrics for the VBA. Finally, Section 8 offers a detailed implementation plan for metrics - our specific recommendations to VBA.

\subsection{TIMELINESS}

Much of the material in this report is based on up-to-date information gleaned from the Applied Software Metrics (ASM) conference held November 10-14, 1991, in Orlando, Florida. Quotes and information taken from informal presentations at the ASM conference are cited in the text by author name only. 


\section{DEFINITIONS OF QUALI'TY}

\subsection{QUALITY IN GENERAL}

For administrative, management, and production activities, quality may be defined as conformance to requirements (CTR). Put another way, it is "doing it right the first time." These are only two possible ways to describe the meaning of the term quality as it applies to these activities.

The meaning of quality as used in this document is different from the commonly accepted meaning of quality, which is strongly related to price. For example, if you require transportation to and from work and shopping, an economically priced vehicle will satisfy that requirement. A luxurious automobile will also satisfy the requirement, but at a much higher price. Thus, the luxurious car, even though it is thought of as a "quality" vehicle, is not justified for the quality requirement as used in this document.

\subsection{QUALITY WITH RESPECT TO SOFTWARE}

The term quality is somewhat different when it is applied to software than when it is applied to administrative, management, and production activities. The global requirements of quality listed above need to be clarified and slightly revised before being applied to software. CTR still applies, since software must do what is required of it. On the other hand, "doing it right the first time" rarely applies to software quality, because software requirements change frequently and can be expected to change throughout the life cycle of the software system.

In addition to CT'R, software quality is further defined as "fitness for use." This definition encompasses all those features recognized as being beneficial to users. Another applicable definition is as follows: "Software that combines the characteristics of low defect rates and high user satisfaction" (Capers Jones). Yet another definition of quality is this: "The totality of features and characteristics that bear on its ability to satisfy stated or implied needs" (Fletcher Buckley).

Quality is necessary (but not sufficient) to achieve reliability and program and data safety. Thus, the definition of software quality implies all of the acceptable definitions given in this section; the definition will necessarily be expanded to fit the particular needs of each situation. The words of Watts Humphrey" summarize the problem of software quality and metrics very well: "If you don't know where you're going, any road will do. If you don't know where you are, a map won't help."

\footnotetext{
"Humphrey is a well-known expert in the field of software quality. The source of this quoted material is not known.
} 
To determine quantitatively whether a software/hardware project meets the criteria given in the preceding paragraphs, a software metrics program must be undertaken. Such a program requires that sufficient management control be exercised over the entire software project, including management, software, and hardware. Some necessary management controls are described in the following section. 


\section{REQUIREMENTS FOR SUCCESSFUL METRICS IMPLEMENTATION}

\subsection{GENERAL REQUTREMENTS}

For a metrics program to succeed, the corporate environment must provide management controls over the software and staff so that the meaningful metrics may be acquired. Any software system utilized by more than one user must be subject to strict management controls. It is critically important that any changes be made in a logical and consistent manner. Thus, quality control, quality assurance, and configuration management are extremely important. To verify that these controls are effective, software metrics are needed to provide quantitative information on software quality.

Approved versions of design documents, code versions, error fix requests, and design change requests must be controlled so that multiple versions do not hopelessly complicate the metrics process. VBA should determine whether implementation of its SDLC is sufficient to permit application of metrics.

To achieve all these desirable goals, there must be total commitment by top-level managers. They must be committed to unilaterally imposing software control and to reporting problems and defects. Management must enforce compliance by system designers, software personnel, and system users. Individuals and groups concerned with monitoring quality should report to top management and be independent of all other organizational groups.

In addition, users must be given the opportunity to participate in formulating the implementation process. Users must be made to feel that their opinions will be given a thorough and fair evaluation. It is probably worthwhile to have users provide their comments and concerns in writing, to have the response in writing, and to make a collection of the user-supplied documents and staff replies available to all interested parties.

\subsection{STAFFING CONSIDERATIONS}

When the necessary management controls are in place, the next consideration is staffing. To achieve quality in an effort that is large in scope and to schedule the effort successfully, staff members who are committed to quality are important; they will determine the success or failure of the project. It is often said that $90 \%$ of the problem in completing a project is in the people themselves. Without adequate staff and management of that staff, the project will fail. Some important factors are considered below; the list of factors is by no means complete because it is not within the scope of this report to make it so.

\subsubsection{Time and Phase of Project}

The number of staff members participating in a development and/or maintenance project varies with time or phase. Adequate planning for each phase will help to ensure full 
staff utilization. Figure 1 shows staffing requirements as a function of time and SDLC phase. It illustrates the varying staffing requirements for each phase of the life cycle; it also points out that different staff capabilities are needed in each phase, although there is some overlap. The figure is adapted from the presentation of Larry Putnam.

\subsubsection{Management of Personnel}

"People priblems" encountered during metrics implementation include these:

- Inability in getting people to track the data,

- Uncertainty as to whether other projects are reported on consistently,

- Middle-level management resistance (i.e., concern over extra overhead costs and the use of the data in assessing management's performance),

- Fear of cultural change, and

- Lack of expertise on how to use metrics.

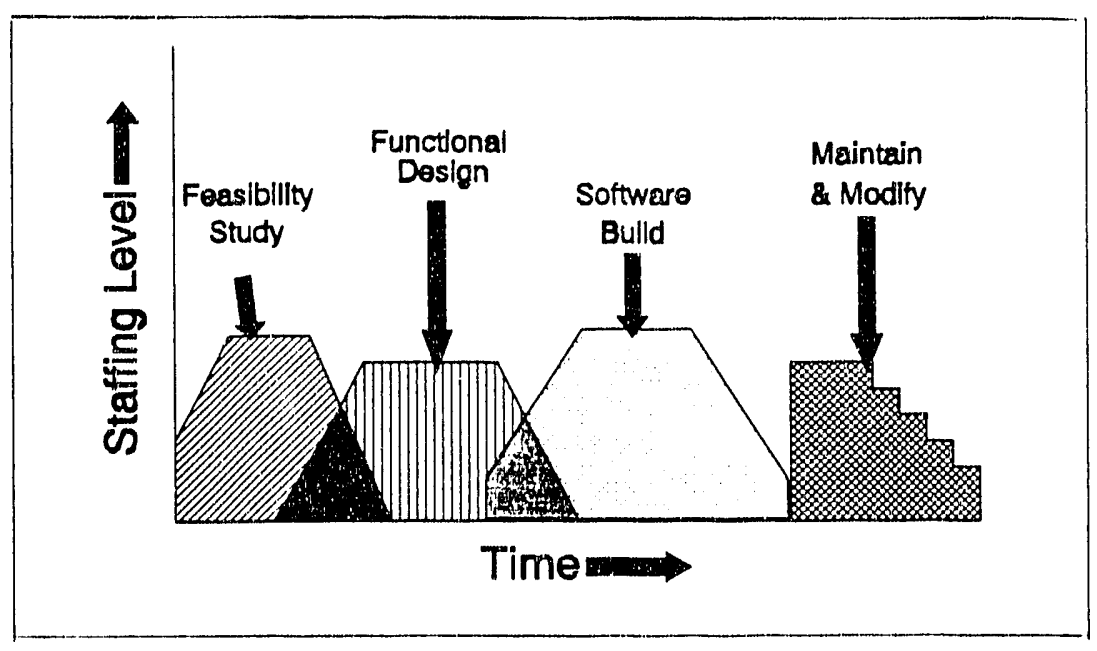

FIGURE 1 Staffing Level as a Function of Project Phase and Time (Source: Adapted from L.H. Putnam) 


\section{FACTORS THAT REVEAL SOFTWARE QUALITY}

When proper management and staffing are achieved, the factors (or attributes) by which quality is measured are of immediate concern. Software metrics are then used to provide information about these factors. Following is a list of quality factors that reveal CTR, doing it right the first time, and fitness for use. The types of measurements (metrics) used to provide information on these factors are described in Section 5.

- Correctness. Results of any information processing must be correct in all significant aspects.

- Efficiency. System response time must be acceptably short and not demand excessive computer resources.

- Flexibility. Information systems always involve change and must be flexible enough to enable implementation of these changes in a reasonable time period.

- Integrity. Information must be consistent and noncontradictory.

- Interoperability. Information systems must be able to operate simultaneously on a variety of hardware and software platforms.

- Maintainability. Hardware and software systems must be maintainable by personnel who might change in response to changing software requirements and changing hardware availability. If code is maintainable, only a minimum number of side effects should result from any modification.

- Portability. Software must be able to move easily to new operating systems and/or new hardware platforms.

- Reliability. Information systems must perform in a reliable fashion and not be subject to idiosyncracies.

- Reusability. Some portions of software can be written so that they are not specific to the hardware and/or software at hand. These portions may tisen be used later in other information systems.

- Testability. All software should be capable of being tested to demonstrate that it performs correctly and does not cause unexpected results in other parts of the information system.

- Usability. Software must be designed with ease of use as a major requirement. 
- Economy. Software goals must be achievable at a reasonable, predictable cost.

- Adequacy of documentation. Documentation must be adequate to support future maintenance and modification.

- Understandability. Code should be written so that others can understand it, so they can determine what it actually does or modify it.

- Flexibility of code. Chaotic times require that code be flexible enough to meet changing requirements. 


\section{SOFTWARE QUALITY METRICS}

\subsection{INTRODUCTION}

This section of the report provides an overview of available software metrics that can be interpreted to yield information about the software quality factors described in the previous section. Not all of the quality factors listed in Section 4 are directly measurable. This section lists what can be measured." The list is quite general and describes metrics commonly used in industry and business, both for business applications and technical software. Not all of these methods will be needed for the VBA metrics program.

According to Schneidewind, "The purpose of software quality metrics is to make assessments throughout the software life cycle as to whether the software quality measurements are being met. . . The use of software quality metrics within an organization or project is expected to have a beneficial effect by making software quality more visible." David Card" puts it this way: "The purpose of measurement in an industrial organization is to provide information that improves decision-making in time to effect the outcome of the process."

According to Mike Daskalantonakis, metrics should be:

- Simple to understand,

- Precisely defined,

- As objective as possible,

- Inexpensive to use, and

- Robust.

Metrics must be simple so that they can be understood by all concerned, from the corporate executive officer (CEO) to the programmers to the users. To achieve simplicity, precise definition is necessary. One metric, for example, is the number of source lines of code (SLOC), which is sometimes expressed in thousands of lines of code (KSLOC or KLOC) or sometimes in just lines of code (LOC). Most metrics users count only certain lines, excluding comment lines and specification statements but including procedural statements and data

'Card, D.N., and R.L. Glass, 1990, Measuring Software Design Quality, Prentice-Hall, Inc., Englewood Cliffs, N.J.

'Schneidewind, N.F., 1991, Report on the IEEE Standard for a Software Quality Metrics Methodology (Draft) P1061, with Discussion of Metrics Validation, in Proceedings of the Fourth Software Engineering Standards Application Workshop, Institute of Electrical and Electronic Engineers, San Diego, Calif., May. 
definitions. To compare SLOC when different code packages are written in different source languages, Daskalantonakis recommends converting source coding into assembly language, because the number of LOC needed to accomplish any given task varies considerably from one source language to another, whereas the number of lines of assembly language code should be about the same, no matter which high-level language is used to code the task.

Metrics should also be objective so that there is minimal subjective interpretation by those who measure and by those who interpret the metrics. If the metrics are expensive, they may not be worth the cost. As for robustness, metrics should apply without failure to most of the system code.

Metrics should be applied tr all phases of the life cycle. Several metrics will need to be applied, because each metric focuses on a single quality factor. To be valid, rnewics rnust be closely related to the quality factor they seek to measure. A very important conclusion is given by Daskalantonakis: "Remember: Meisurement is not the goal. The goal is improvement through measurement, analysis, and feedback."

Problems and nonconformances can be measured in a number of ways, as indicated in the next section. These neasurements are used to monitor overall systern performance and to evaluate the effectiveness of system management, system design, and quality control measures. They also provide a measure of the timeliness of corrective procedures.

Software metrics are sometimes classified according to phases of the software system life cycle. If the software system for VBA were just beginning (i.e., in the design stage or earlier), it would be appropriate to organize the material by life-cycle phase. For clarity here, however, software metrics are presented according to their separate functions.

\subsection{RESOURCE (COST) METRICS}

Cost is always an important consideration in software engineering efforts. The importance of any single cost metric varies from one environment to another, depending at least in part on resource availability. Cost metrics are as follows:

1. Number of effort hours expended

a. Management support

1) Top management

2) Middle management

3) Line management

b. Technical support,
1) Design
2) Implementation
3) Testing 
4) User support

5) Rework

2. Maintenance of schedules (number of weeks for initial construction or major modifications)

a. Predicted

b. Elapsed

\subsection{SOFTWARE DESIGN METRICS}

In most cases, more than $50 \%$ of software problems and nonconformances are caused by software '- $r$ sign errors. In the preliminary design phase, two major tasks are undertaken: defining the job that the softwarf must perform and generating a first design concept, which states how the sof? ware should be structured to perform the job. Components of this preliminar: phase include defining system requirements and software requirements, establishing design specifications and technical suftware specifications, analyzing and allocating software requirements, analyzing the operational sequence, and establishing verification requirements. Other important tasks include designing databases and creating preliminary design phase documentation.

Following the preliminary design phase is the detailed design phase, in which the preliminary design of the computer programs is reviewed and the design is completed. The preliminary design is analyzed to demonstrate that all design requirements have been met. This analysis includes studies of the interface design, software modeling, software operation design, and detailed software design. Data flow diagrams may ke useful at this stage of development. The detailed design phase must be thoroughly documented; documentation should include functional descriptions, subroutine usage identification, and descriptions of inputs, processing methods, limitations, and the software testing methodology.

Software design metrics are as follows:

1. Complexity. Important factors in software design are the optimization of module complexity, minimization of data variables, minimization of connections, and use of modern programming techniques. Complexity is a very important metric, possibly the single most important metric. The more complex a software system is, the larger is its error rate. Card has established a relationship between errors and design complexity (see Figure 2). Complexity, as computed by using formulas given by Card, is determined on the basis of number of input/output variables, fanout (described below), and number of modules in the system.

The relation ship between module size and complexity according to Card is shown in Figure 3. Apparently, there is an optimal number of 


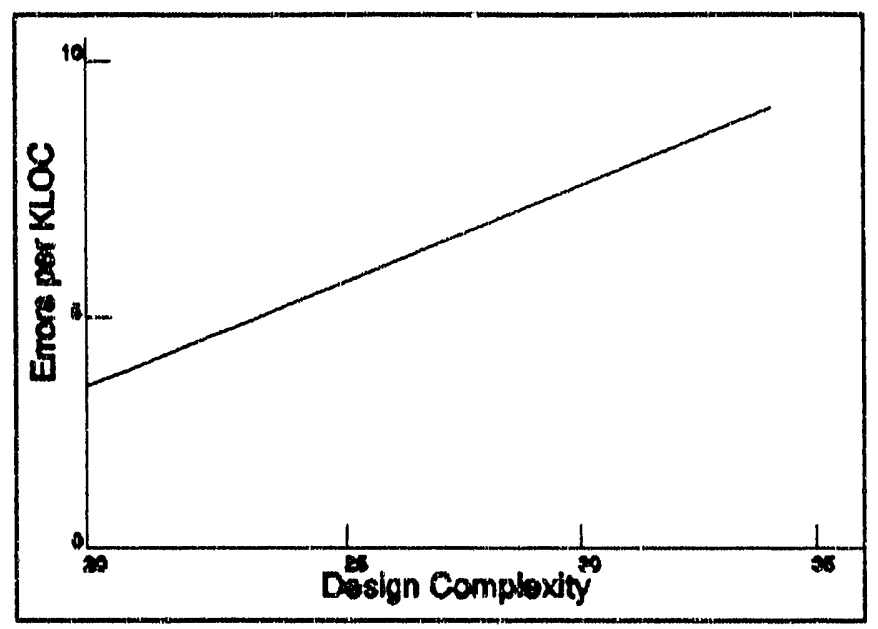

FIGURE 2 Error Rate as a Function of Software Systen Complexity (Source: Adapted from D. Card)

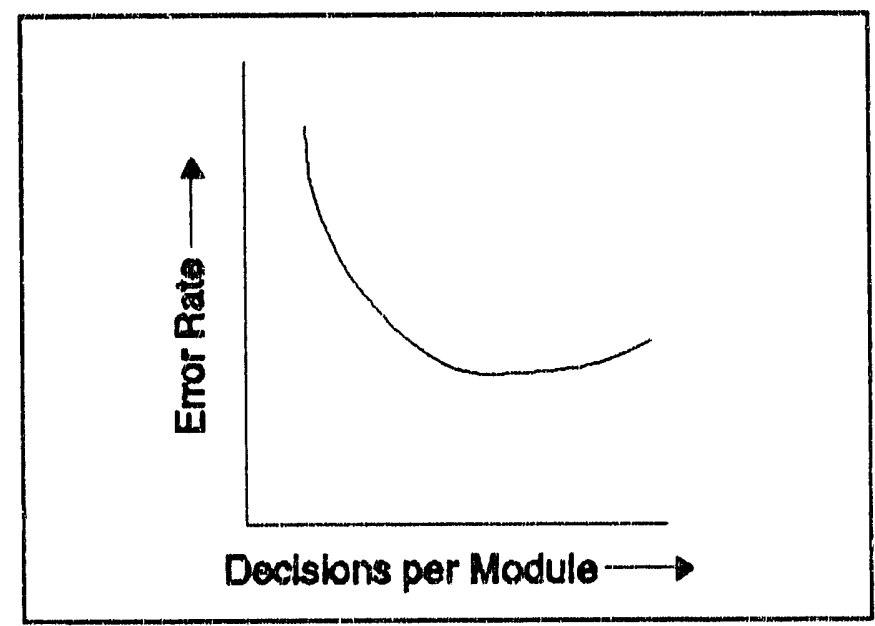

FIGURE 3 Error Rate as a Function of Module Size (Source: Adapted from D. Card)

decisions per module for which the error rate is at a minimum. This effect may possibly illustrate that if too few decisions per module are made, more modules will be necessary to process all the decisions, which implies a need for substantially more LOC. At the other extreme, if too many decisions per module are made, the modules will become longer and harder to maintain.

The advantage of the complexity metric is that it can point to unnecessarily complex coding and possibly lead to more straightforward coding, thereby reducing the chance for logic errors. However, too much emphasis on reducing complexity can be counterproductive; in 
cases where software must deal with comples situations, the software itself must necessarily be corrplex. Components of the complexity metric are as follows:

a. Functional allocation.

1) Number of functions/subsystems. The complexity of a software system is directly proportional to the number of functions it performs or the number of subsystems it requires. In other words, the greater the number of different operations a system can perform, the more complex it is likely to be.

2) Number of data sets/interfaces. Also contributing to the complexity is the number of data sets or databases the system must use and the number of interfaces between hardware components.

b. System design complexity.

1) Number of variables. The greater the number of variables there are in a system, the greater is its complexity. This problem is not necessarily overcome by using variables for more than one purpose.

2) Number of units. In addition, more complex systems use more units or modules.

3) $\Sigma$ (fanout squared). Fanout is the number of calls or transfers to other modules from a given module. Thus, if each of two modules calls two other modules, the $\Sigma$ (fanout squared) $=2^{2}+$ $2^{2}=8$; however, if cne module calls one module and the other calls three, this metric is larger $\left(1^{2}+3^{2}=10\right)$, resulting in greater complexity.

2. Satisfaction of requirements. The percentage of functional and performance requirements that are met provides a straightforward measure of software quality. This important metric provides a good measure of design quality. It becomes less important as the end of the life cycle is approached. The number of design changes can provide information about how well a software system satisfies requirements. Too many design changes may indicate that the initial phases of the life cycle received insufficient attention. However, a large number of design changes could also have been caused by rapidly changing requirements. 
3. Conformance to standards. The total conformance of a program to detailed standaris is not recomriended; rather, a few guiding principles should be applied as appropriate. Over-reliance on standards may severely limit productivity. For the software to comply to interoperability and transport requirements, programmers should avoid using system-dependent coding and procedures as much as possible. Initially there may be some nonconformance to standards. The number of nonconformances gives an indication of how much the software system deviates from defined standards for coding. This number should not be allowed to increase.

\subsection{SOFTWARE PRODUCT METRICS FOR CODE BEING MAINTAINED}

The software product is what results from coding the system as designed. The product is usually used, but it may or may not be satisfactory. Software product metrics are as follows:

1. Quality. The number of software errors detected during system integration and testing is used to measure design and coding quality. This is a very useful metric at this stage of the life cycle. The number of design defects shows how well the system was designed.

a. Number of software errors. Software errors are discovered when the system is shown to produce the wrong result or action as defined by the system design. The number of software errors is determined from the problem reporting system (see below).

b. Number of design defects. When the system does not provide what is required, the problem may be traceable to a failure in the system design. This failure may have been caused by an oversight on the part of the designers, an omission that was not thought of by those preparing the specifications or design, or an omission resulting from the fact that the users may not have realized that they would want this missing capability.

2. Volume. These unit (or module) numbers are related to the effectiveness of the system modification. This metric is primarily useful during initial coding and system modification phases.

a. Number of new units needed. This is the number of new units (or modules) ileeded to provide functionality as perceived during use of the software product. A large number of new units needed may mean that engineering done early in the life cycle was poor. 
b. Number of modified units required for design change. The number of existing units that must be modified to anhieve the required results and functionality is again a measure of the success of the engineering in the specification preparation phase or the design phase.

c. Number of reusable units that can reduce the coding effort. Reusable modules (units) save coding effort; hence, the number of reusable modules is positive if it represents a significant fraction of the total number of units.

d. Function points. Function points provide a measure of discrete actions provided by the code. Function points are calculated from the numbers of inputs, outputs, inquiries, data files, and interfaces. The value of function points as metrics is questioned by many experts in the field. Calculation of function points is complicated and subjective, and many of those who have used them have discarded them. Because of the lack of general agreement on function points, their complexity, and their subjectivity, they are not discussed further in this report.

3. Documentation. The documentation should be complete and thorough without being excessive and verbose. This is another important metric.

a. Number of documentation pages or words. The number of words in the documentation is probably the best measure of the amount of documentation, since the number of pages may be padded by blank or partially blank pages. Documentation must be sufficient to provide thorough descriptions of the coding, variables, inputs, files, etc.

b. Number of errors found in the documentation. Lengthy documentation is certain to contain many errors. Staff should be assigned to revising the documentation so that each documentation release contains fewer errors than the previous one. A count of the nur.zber of errors found in each release will demonstrate whether dociruentation is or is not improving.

c. Number of errors and problems caused by incorrect documentation or by documentation that is not up to date.

\subsection{SOFTWARE TESTING METRICS}

Software should be tested through the use of as many possible variations on input and output as possible. Because the software testing phase is often inadequate or completely missing, this metric is very important. The number of code modules tested is a very useful metric. 


\section{CASE STUDIES}

Actual case studies of successful applications of software metrics are presented in this section.

\subsection{STUDY 1}

A large, multinational corporation had been in the initial phases of design and functional specification preparation. The former was nearly complete and the latter had assumed mammoth proportions (thousands of pages). The CEO was expecting delivery of the completed product in a few months. There had been minimal user involvement in the design phase, and little actual coding had been completed. With little senior management and support, the project appeared to be on the brink of failure. Consultants were brought in. They reorganized the entire project, modified goals, established a schedule, and established an ongoing metrics program. Metrics used included KLOC, software errors, and scheduling. Because morale was so low, the consultants also monitored morale, but they did not say how they measured it. The project is now on track, with delivery of the most important subsystem scheduled for delivery in the near future. A major key to this turnaround was the implementation of metrics, which determined where progress was good and where it was not sufficient.

\subsection{STUDY 2}

A very large financial organization sought to improve its competitive position and improve profitability. One of the keys to realizing these goals was through extensive computerization. The project had been underway for several years with unimpressive results; most of the software packages were still on the drawing board. The morale of the technical staff was very poor, and staff turnover rate was very high. The CEO wanted his software immediately. Consultants were hired. They reorganized the entire project, added computeraided software (CASE) tools, and established a metrics program. They also persuaded the CEO to relax his demands and make more realistic ones. The result was that managers had the metrics to quantitatively track the progress of the newly established demands, and measurable progress was made on a realistic schedule. The metrics used were not specified.

\subsection{STUDY 3}

Another financial institution implemented a metrics program and determined that it cost $\$ 0.28$ per line of COBOL code per year to maintain the code. Metrics used included KLOC (expressed as maintenance time per KLOC). The metrics data pointed to problem areas previously unsuspected. As a result, corrective actions were taken. In the end, maintenance costs were reduced to $\$ 0.01$ per line of code per year, representing a reduction by a factor of 28 . 


\subsection{STUDY 4}

A well-known manufacturing company perceived the need for a metrics program to gain control over software costs. Many metrics were used; a few of the more important ones included software errors, design defects, and scheduling. The goals were to quantify the errors and defects in the software life cycle, quantify the rework effort (cost) associated with these errors and defects, and prioritize the company's investment by quantifying the performance of fault-containment and fault-prevention activities.

Benefits realized to date include these:

- Engineers and managers started thinking about the software process and quality.

- A new understanding of problems was achieved.

- The focus was on actions with quantitative results.

- In some cases, quality improved by a factor of 50 .

- Many indirect benefits (for example, improved ship-acceptance criteria) were achieved.

Long-range benefits expected include these:

- Software groups will learn from previous mistakes and take action to prevent their recurrence.

- Significant impiovements in customer satisfaction will be achieved.

- Costs will be reduced significantly, and resources will be freed for development of new software.

- Improvements in productivity will reduce the amount of time it takes for new products to reach the market. 


\section{SUGGESTED METRICS TO BENEFIT THE VETERANS BENEFITS ADMINISTRATION}

\subsection{CANDIDATE METRICS}

The actual metrics implemented by VBA will be determined by VBA staff and management. Those listed below are candidates for possible incorporation into VBA's plan.

\subsubsection{Software Design Metrics}

1. Satisfaction of requirements: Number of design changes. All system changes should be documented thoroughly, including the date the change request was approved. The frequency of design change approvals (e.g., changes per month) should then be plotted as a function of time. A declining frequency with time is indicative of satisfactory design. A plot of design change frequency versus time would be most useful in quantifying system adequacy.

2. Conformance to standards: Number of conformances. Violations of standards employed by VBA should also be documented and plotted as a function of time. A sizeable number of nonconformances is indicative of poor system design.

3. Complexity. The importance of complexity has been discussed. In addition to those sources already mentioned, Figliulio pointed out the applicability of the McCabe Cyclomatic Compumetric for COBOL programs. For a COBOL code of 280 lines, a metric of 10 is labeled good, 30 is difficult, 50 is untestable, and 75 is unmaintainable. The metric is computed by counting the number of program ifs, ands, ors, nots, and statement labels. Elses are not counted. According to Figliulo, this metric is the most important metric of all.

\subsubsection{Software Product Metrics}

1. Quality: Number of software errors (or software coding errors). Measuring the frequency of software errors provides a good indication of design quality. A decline in the frequency over a period of time indicates a maturing software system that is operating under good lifecycle control methods and procedures.

2. Documentation. The volume of documentation (pages, lines, or words) provides a measure of the extent and adequacy of the documentation. Any production software system should be thoroughly documented. In addition to volume, software documentation needs to be inspected for 
clarity and completeness - a more subjective approach not easily amenable to metrics.

\subsubsection{Software Testing Metrics}

1. Number of code modules test, $l$. An important metric is the portion of the number of code branches that have been adequately tested. The ideal metric should be close to $100 \%$.

\subsubsection{Resource Metrics}

1. Technical support: Rework. The number of rework hours spent by technical personnel is a very important metric. Measured as a function of time after code system acceptance, this metric should decline with time until a low steady state is reached.

2. Management support. The number of management support hours should show a similar decline to a low value. Too much management involvement suggests a real need for management reorganization.

3. Technical support: User support. The number of hours required for user support should diminish with time but may increase when substantial modifications are made. User support is defined as the number of hours spent by the software staff to assist users in realizing their goals plus any time devoted to teaching users how to use the system effectively by providing training classes.

4. Technical support: Enhancement. The number of hours required to modify the software will reveal insights into design adequacy; a plot of this metric versus time should indicate that few hours are expended except during substantial code modification.

5. Technical support: Conversion. The labor expended in converting the system or components of the system on different hardware and/or software platforms provides a good indication of the design quality. If too much time is spent on conversions, initial designs were too dependent on hardware/software.

6. Maintenance of schedules. The ratio of the number of deliverables presented on schedule to the total number of deliverables provides an indication of the quality of management control over the software development and rework stáf. 


\subsection{PROCESSING THE ACQUIRED METRIC DATA}

\subsubsection{Step 1: Analyze the Metric Data}

Metrics provide data that must be carefully interpreted to extract the meaning of each metric. These meanings must then be synthesized into a cohesive picture of the automated data process. From this picture, conclusions should be drawn as to which parts of the system (including personnel) are performing well and which need attention and modification.

\subsubsection{Step 2: Validate the Analysis}

The entire metric process, from data collection to interpretation, should be reviewed to determine whether the metrics are providing a reasonable estimate of software quality (Section 3 has a disc'sssion of software quality). Often the metric process will need to be revised to improve the quality of the analysis. Individual metrics may not be providing the desired information. Management needs may not be adequately met by the metrics process, and modifications may be required to provide better answers. 


\section{IMPLEMENTATION PLAN}

We recommend that the VBA consider implementing its metrics in a step-by-step fashion, beginning with the first step listed below and proceeding through the remaining steps in the order presented. It is clear that VBA staff members are in the best position to choose a potentially productive order. It is not necessary to complete any given step before starting the next step. The order of the steps was chosen so that the VBA can derive the most from the program as early as possible.

1. Step 1: Develop an implementation plan. The first step is to develop a detailed implementation plan for presentation to top management for approval. It may be advisable to create a preliminary version of the plan first, get suggestions from management and users, and then generate a final version.

2. Step 2: Begin implementation of a configuration management program. As indicated above, a configuration management program should be implemented and enforced. Metrics will mean little if the code is changed frequently.

3. Step 3: Secure top management's support. Top management's support is vital to the success of the VBA metrics program.

4. Step 4: Name a metrics manager. The metrics program manager must have the authority to ensure that managers and programmers adhere to the policies given in the metrics implementation plan. The manager should be immune to pressures to skew or falsify the metrics. Thus, he probably should report directly to the CEO.

5. Step 5: Establish a computerized problem reporting system. Create a computerized form containing pertinent information about errors and defects. Table 1 illustrates such a form; VBA should adopt a similar form. These forms are to be filled out on the computer and stored in a database. Once in the database, the initial contents of the form should be immutable. Dated additions to the form may be made upon permission of the metrics program manager. Information on the resolution of any problem should be added to the report when the problem is solved. Copies of the report are to be distributed as appropriate.

6. Step 6: Establish a design change management system. Design change requests are larger in scope than error reports; their ultimate purpose is to extend the capability of the system to accommodate new requirements or remedy design defects. Design change requests, unlike error 
TABLE 1 Suggested Contents for Problem Report

Problem entry number
Product name
Release identifier
Driver identifier
Functional line item
Process stage in which problem was detected
Process stage in which prnhlem was created
Quality Improvement Team Department (for suggestions)
Problem report number for miscellaneous problem suggestions
Date that problem was entered into the system
Person who should be contacted for questions (owner)
Analysis type (problem investigation, test, field, Q/A, miscellaneous)
Problem investigation type (group, peer, team leader, other)
Current status (reported, being investigated, closed)
Closing reason codes, answer text, owner identification, date closed
Category of cause (communications, education new function, education, base function,
other, oversight, transcription prohlem, mistake)
Abstract of problem (short description)
Description of cause cf problem
Assigned process improvement suggestion list
Description of problem
Suggested improvements
Log of all activities related to this problem

Source: Jones, C.I., 1985, IBM SJ, no. 2, pp. 163.164.

problem reports, are likely to be quite lengthy. Like problem reports, however, design change requests must be stored in a database and protected from tinkering. It is suggested that design change reports be approved by top management before implementation is initiated.

\section{Step 7: Implement the first and most important metrics.}

a. Number of software errors (see Section 7.1.2, Item 1). This metric primarily indicates programming errors. It is generated from the database containing the problem report data. The number of problem reports per unit of time is recorded. The VBA will have to determine the size of the time unit; as a first trial, it is suggested that the time unit be one month. After three or more months of data collection, the data should be plotted. The plot should show the number of defects per month as a function of time. It will also be useful to plot the number of fixes on the same graph. In general, the value of this metric should decline with time. Some scatter in the data is to be expected, and the rate may rise considerably following large changes such as those resulting from a design change request. Analysis of metric data is to be performed by the metric 
implementation program manager or his or her subordinate. The problem report should contain the number of hours used for the rework. These data should also be plotted, perhaps on the same graph.

b. Number of design changes (see Section 7.1.1, Item 1). This metric primarily shows design errors, oversights, or deficiencies. It is prepared from the database containing the design change requests. The method of analyzing the data is similar to that used for the software error metric. Design change requests are plotted as a function of time. Analysis and plotting of the data are to be performed by the metrics manager or his or her designated staff member.

c. Complexity (see Section 7.1.1, Item 3). Although the consensus of the experts is that complexity is the most significant metric, it has not been suggested until now because of the work involved. For a COBOL code of 100 lines (procedure division only), a metric of about 3.5 is satisfactory, 11 is difficult to maintain, 18 is untestable (i.e., so complex that no satisfactory test could be devised in a realistic time period), and 27 is unmaintainable and should be replaced with newer, simpler code. These numbers should not be considered unalterable; they need to be interpreted correctly. The metric is computed by counting the number of program ifs, ands, ors, nots, and statement labels, and dividing the number of lines of code in units of 100 lines. Elses are not counted.

The interpretation of this metric is as follows. Metrics are tabulated for each module. All modules in the code having the greatest number of complex metrics, or those modules having more than 15 17 metrics, should be rewritten in a much simpler fashion unless they are never to be used again. Modules with the greatest number of complex metrics are top candidates for being rewritten since they are most likely to cause errors. The complexity metric, together with the software errors metric, is most useful in locating troublesome modules and coding.

8. Step 8: Implement other metrics later.

a. Documentation (see Section 7.1.2, Item 2). The volume of documentation should be charted as a function of time. The number of words in the documentation appears to be the most reliable source of data for the metric. In general, the volume metric should increase with time, although decreases may be experienced when pcrtions of the system are excised. If possible, documentation errors and omissions should also be tracked. 
b. Management support (see Section 7.1.4, Item 2). The nurnber of topmanagement hours consumed in support of the system and in remedying defects or other problems should be tracked as a function of time. A marked increase in this metric may be cause for concern, although increases resulting from major system changes may be expected. A plot of management hours versus time is to be prepared. It seems very probable that top manugement's involvement will be at a maximum at the beginning of the project and when major changes in direction are considered.

c. User support (see Section 7.1.4, Item 3). Users of any substantial computer software system will need the support of system analysts and programmers. Training for users nust be provided by computer staff. The hours devoted to user support should be tracked. Excessive user support hours may indicate insufficient or inadequate training; a system rework to improve usability (i.e., user friendliness) may be needed.

d. Enhancement (see Section 7.1.4, Item 4). The cost to enhance the software system should also be tracked. Eircessive enhancement costs may be caused by poor documentation, which implies a need for effort to improve the docurneritation. Excessive enhancement costs may also be traceable to design inadequacies, and no cost-effective means to repair the design may be available. For this metric, costs are recorded for each majol change and serve as basis for future decisions.

9. Step 9: Implement additional metrics if necessary (see Section 7.2). Annual reviews should be corvened to determine the efficacy of the metrics program, check for the existence of problem areas for which no metrics are currently in place, and implement new metrics if necessary. Many other metrics are known, and their utility should be consiclered. 

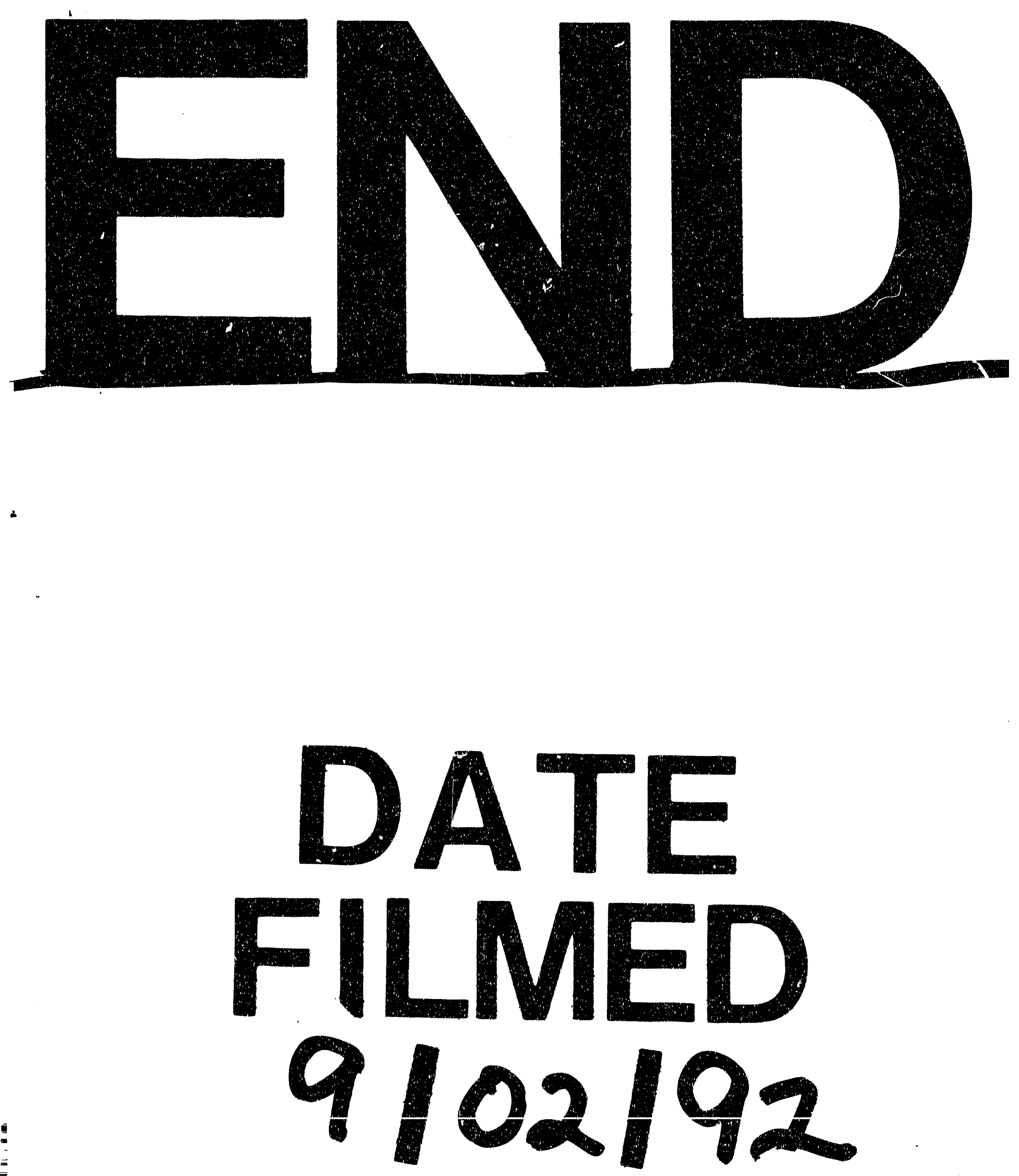
1 